\title{
An accurate determination of the electronic transitions of InAs/InGaAs/InP quantum dots for midinfrared lasers using simultaneous complementary spectroscopic techniques
}

\author{
T. K. Sharma, ${ }^{1, a)}$ T. J. C. Hosea, ${ }^{1, b)}$ S. J. Sweeney, ${ }^{1,2, c)}$ and X. Tang ${ }^{3}$ \\ ${ }^{1}$ Department of Physics, University of Surrey, Guildford, Surrey, GU2 7XH, United Kingdom \\ ${ }^{2}$ Advanced Technology Institute, University of Surrey, Guildford, Surrey, GU2 7XH, United Kingdom \\ ${ }^{3}$ Photonics Research Centre, Microelectronics Division, School of Electrical and Electronic Engineering, \\ Nanyang Technological University, 639798 Singapore, Singapore
}

(Received 5 August 2008; accepted 4 September 2008; published online 31 October 2008)

\begin{abstract}
InAs/InGaAs/InP quantum dots (QDs) emitting at $\sim 2 \mu \mathrm{m}$ for midinfrared laser applications are studied using the complementary spectroscopic techniques of photoluminescence (PL), photoreflectance (PR), and surface photovoltage spectroscopy (SPS). We use a procedure that ensures that the same sample spot is studied virtually simultaneously by these three different spectroscopic techniques under almost identical conditions. We are able to measure the ground and excited states transitions of the InAs QDs without any ambiguity, thus providing a complete and clear understanding of the electronic transitions. Temperature dependent PL, SPS, and PR measurements provide a systematic thermal evolution of the ground and excited states. However, the QD transitions are not all seen together at any given temperature in either the PL or SPS measurements. By contrast, the PR technique can measure the complete set of ground and two excited state QD transitions and also the InGaAs barrier energy at all studied temperatures. (C) 2008 American Institute of Physics. [DOI: 10.1063/1.3005903]
\end{abstract}

\section{INTRODUCTION}

Due to its simplicity and power, photoluminescence (PL) is generally the most common technique applied to investigate quantum-confined structures. However, PL often fails to give a strong signal due to defects/dislocations, especially in quantum dot (QD) structures grown using StranskiKrastanov (SK) procedure. Even if PL is possible, it may not reveal all the electronic QD transitions because several may have very poor radiative efficiencies. It is therefore essential to have available complementary spectroscopic techniques such as photoluminescence excitation (PLE), photoreflectance (PR), and surface photovoltage spectroscopy (SPS), where the latter essentially measures the absorption spectrum of the quantum structures. Chouaib et al. ${ }^{1}$ implemented PL, PLE, and PR spectroscopic techniques to study InAs/InP QDs emitting at $1.55 \mu \mathrm{m}$. Salem et $_{\text {al. }}{ }^{2}$ used PL and PLE to explore the excited states (ESs) in InAs/InP QDs. However, all these complementary spectroscopic measurements were performed under different experimental arrangements where it was therefore difficult to ensure that the same sample spot was investigated. This severely limits the usefulness of such complementary spectroscopies of SK-grown QDs because these material systems are inherently spatially inhomogeneous and it is therefore very important to map the same sample spot when using different measurement techniques.

\footnotetext{
a) Author to whom correspondence should be addressed. Electronic mail: tarun@ rrcat.gov.in. Present address: Raja Ramanna Centre for Advanced Technology, Indore-452 103, India.

${ }^{b)}$ Electronic mail: j.hosea@surrey.ac.uk.

${ }^{c}$ Electronic mail: s.sweeney@surrey.ac.uk. Currently on leave at Central Technology Laboratory, Philipps-Universität Marburg, Material Sciences Center, Hans-Meerwein-Straße, 35032 Marburg/Lahn, Germany.
}

Here, we present an accurate determination of the electronic transitions of SK-grown InAs/InGaAs/InP QDs by performing virtually simultaneous complementary spectroscopic measurements on the same sample spot. InAs/InGaAs/InP QDs are very important for midinfrared laser applications. ${ }^{3}$ Midinfrared semiconductor lasers operating in the 2-5 $\mu \mathrm{m}$ wavelength range are of special interest for several industrial, biomedical, environmental monitoring, and military applications. ${ }^{4}$ QDs formed with binary compounds of InAs and InSb, or their ternary alloys such as InAsSb and InGaAs have also been explored for mid-IR applications. These QDs are grown on InP substrates because of various advantages as described by Yin et al. ${ }^{4}$ In this article, we present results on InAs/InGaAs/InP QDs using PL, PR, and SPS techniques applied under almost identical conditions.

\section{EXPERIMENTAL DETAILS}

The InAs/InGaAs/InP QDs were grown by metal organic vapor phase epitaxy (MOVPE) with fast nucleation rate and atomic layer epitaxy (two step growth method) in pure nitrogen ambient, which provided a narrow dispersion of dot sizes. ${ }^{5}$ The sample structure details are given in Table I. These QDs are grown with graded strained InGaAs barriers on either side. Since the nominal thickness of InGaAs barrier on either side is less than the critical layer thickness, the in-plane lattice constant matches that of the InP substrate and will therefore provide a strained InGaAs barrier to InAs QDs. ${ }^{5}$ It should be noted that the InAs QDs grown on an InP substrate normally emit at about $1.55 \mu \mathrm{m}$ at room temperature. ${ }^{1}$ In order to extend the emission wavelength of InAs QDs toward the midinfrared range, the QD confinement energy can be reduced by sandwiching them between graded 
TABLE I. Layer structure details for the InAs/InGaAs/InP QD sample investigated in the present work. The nominal thickness and composition of InGaAs barrier layer is given in the table. The indium content of the barrier layer increases from 0.53 to 0.72 when one approaches the QD layer from either side, as shown in the table.

$$
\begin{gathered}
35-40 \mathrm{~nm} \mathrm{In}_{0.72-0.53} \mathrm{Ga}_{0.28-0.47} \mathrm{As} \\
2.5 \times 10^{10} \mathrm{~cm}^{-2} \mathrm{InAs} \mathrm{QDs} \\
25-30 \mathrm{~nm} \mathrm{In}_{0.53-0.72} \mathrm{Ga}_{0.47-0.28} \mathrm{As} \\
\text { InP buffer } \\
\text { InP substrate }
\end{gathered}
$$

InGaAs barriers. ${ }^{5}$ Here, the indium content of graded InGaAs barrier layer increases when one approaches the QD plane from either side, ${ }^{5}$ as shown in Table I, which provides the required reduced energy barrier for carriers confined in the QD layer. The mean dot height and diameter were measured by atomic force microscopy (AFM) for a sample without top barrier and found to be 9 and $54 \mathrm{~nm}$, respectively. Figure 1 shows the three-dimensional (3D) AFM image of an uncapped QD sample. The average QD density is 2.5 $\times 10^{10} \mathrm{~cm}^{-2}$ for the sample studied here.

The PL, PR, and SPS measurements were performed using conventional grating-based spectroscopy and lock-in amplifier techniques. For both the PR and SPS measurements, ${ }^{6-9}$ light from a $100 \mathrm{~W}$ quartz tungsten halogen lamp, located at the primary entrance slit of a multipleport $0.32 \mathrm{~m}$ monochromator system, was dispersed with a 10 $\mathrm{nm}$ band pass and used as the probe beam focused onto the sample surface. All focusing was performed with off-axis parabolic aluminum mirrors.

The SPS measurements were performed by positioning a mechanical chopper $(670 \mathrm{~Hz})$ at the exit slit of the monochromator probe light, before the sample, and using a lock-in amplifier to measure the changes in surface potential induced by the resulting periodic generation and subsequent redistri-

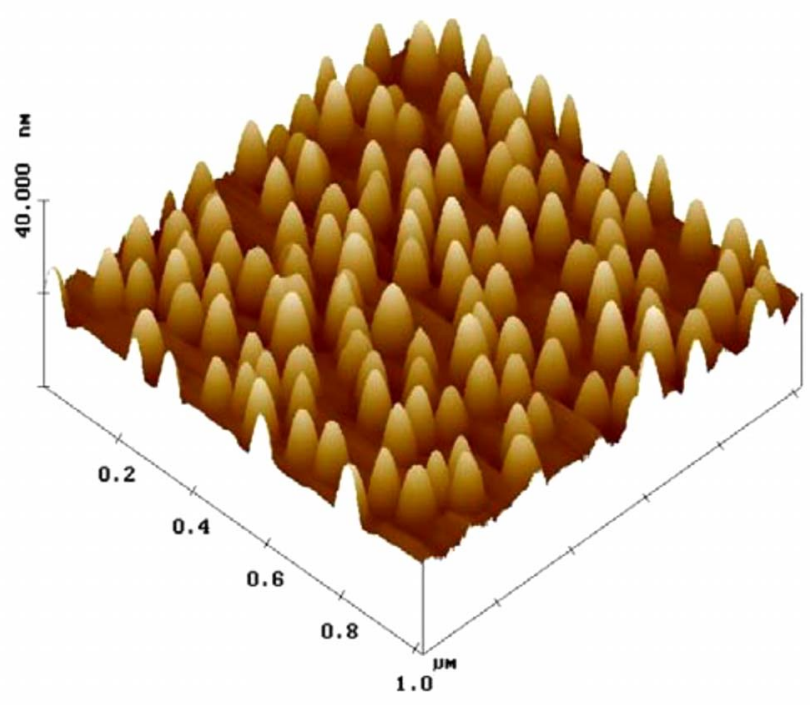

FIG. 1. (Color online) 3D AFM image of uncapped InAs QDs grown on an InGaAs/InP matrix. bution of excess carriers. The small ac voltage was measured in "soft contact" capacitorlike geometry, wherein a transparent conducting glass front electrode was pressed gently against the front surface of the sample, whose back surface was attached with conducting silver paste to a flat grounded copper electrode. ${ }^{10,11}$ The front contact could alternatively be formed more conveniently by attaching a fine copper wire to the front surface of the sample with a small drop of conducting silver paste. In this case, the monochromator light was focused in the vicinity of the small silver drop. The photon flux on the sample was kept low such that the magnitude of the surface photovoltage was also small, i.e., $|\mathrm{SPV}|<$ $<k T / e$, where $k$ is the Boltzmann constant, $e$ is the charge on the electron, and $T$ is the absolute temperature in Kelvin.

For the PR measurements, ${ }^{12}$ the same monochromator probe light as for SPS, only this time with the exit slit chopper halted, was reflected from the sample and focused onto a liquid-nitrogen-cooled $\mathrm{InSb}$ detector to measure the dc sample reflectivity $(R)$. The chopped beam $(810 \mathrm{~Hz})$ of a 100 $\mathrm{mW}$ diode-pumped $\mathrm{Nd}-\mathrm{YVO}_{4}$ pump laser $(\lambda=1.06 \mu \mathrm{m})$ was used to modulate the built-in surface electric field at the same sample spot, and the resulting ac change in the reflectivity $(\Delta R)$ was measured with a lock-in amplifier.

In this PR configuration the same chopped $\mathrm{Nd}-\mathrm{YVO}_{4}$ laser also excited PL (Ref. 13) from the QD sample. Since the monochromator exit slit optics were already configured to focus tungsten emission light onto the sample for the SPS and PR measurements, some PL emission would inevitably be collected back into what is actually the exit slit of the monochromator. Thus the corresponding PL spectrum could be measured using the same monochromator but operated in a "backwards" configuration, and the PL could be detected by another liquid-nitrogen-cooled InSb detector located at a second ancillary entrance slit, which could be easily accessed by moving a small mirror inside the monochromator to select this slit instead of the primary slit where the tungsten lamp was located.

This arrangement thus ensured that the PL, PR, and SPS measurements were performed under almost identical conditions, virtually simultaneously, or at least in quick succession after one another, on the same sample spot, without changing anything except perhaps the laser power (to provide the differing excitation intensities appropriate to the PR and PL) and which chopper was active (chopped probe beam for SPS, or chopped pump beam for PR and PL). In the SPS and PR, the image of the illuminated monochromator slit on the sample was about $2 \times 3 \mathrm{~mm}^{2}$ (sample size $\sim 3 \times 3 \mathrm{~mm}^{2}$ ), while the laser spot was about $1 \mathrm{~mm}$ in diameter. Therefore, the SPS mapped a slightly larger sample area as compared to the PL and PR techniques. To enable the temperature dependent spectroscopic measurements, the QD sample was kept mounted in a closed-cycle helium cryostat with a cylindrical window providing $360^{\circ}$ optical access. The sample was heated in suitable steps from $\sim 9$ to $\sim 200 \mathrm{~K}$ with the three complementary spectra being measured at each temperature before heating up to the next value. The SPS and PL spectra were corrected for the system response. 


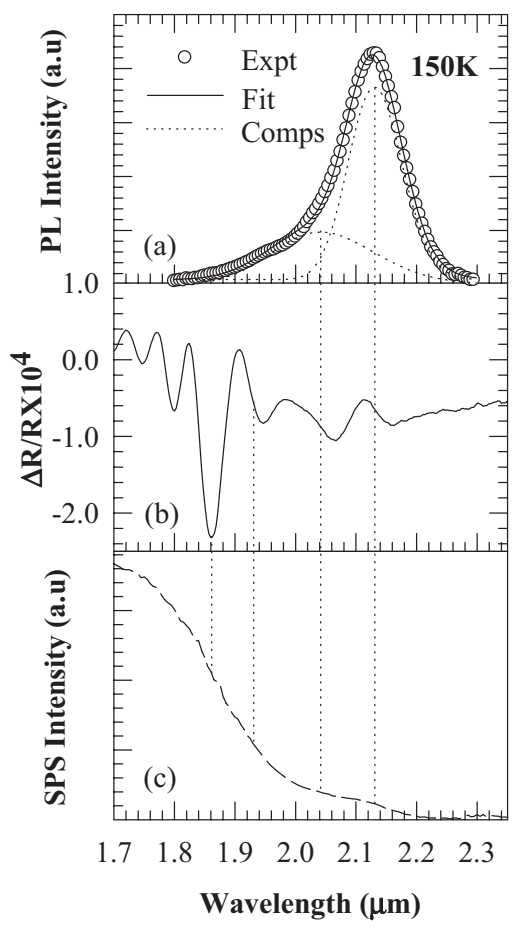

FIG. 2. An example of the virtually simultaneous complementary spectroscopic measurements on the InAs/InGaAs/InP QDs: (a) $150 \mathrm{~K}$ PL spectrum along with a least-square fits (full curve) using two Gaussian components (shown separately by dotted curves), (b) $150 \mathrm{~K} \mathrm{PR}$ spectrum, and (c) $150 \mathrm{~K}$ SPS spectrum. The vertical dotted lines correlate the individual components seen in three spectra.

\section{RESULTS AND DISCUSSION}

Figure 2 shows an example of the virtually simultaneous complementary PL, PR, and SPS spectroscopic measurements performed on InAs/InGaAs/InP QDs at $150 \mathrm{~K}$. Figure 2(a) shows the $150 \mathrm{~K}$ PL spectrum, fitted with two Gaussian peaks (explained in more detail later) representing two electronic transitions associated with the InAs QD structures. These two transitions are also related to the two lowestenergy features seen in the corresponding PR spectrum, as shown schematically in Fig. 2(b) by the two vertical dotted lines at $\sim 2.04$ and $\sim 2.13 \mu \mathrm{m}$. There are several further features seen in the PR spectrum, as shown by the two vertical dotted lines on the shorter wavelength side, near $\sim 1.86$ and $\sim 1.93 \mu \mathrm{m}$, which could be related to the $\mathrm{InAs} / \mathrm{InGaAs} / \mathrm{InP}$ QD structure. The signatures of the transitions seen in the PL and PR may also be discernable in the SPS spectrum in Fig. 2(c), as subtle features at approximately the same wavelengths, such as changes in slope. The identification and origin of these features will be fully elucidated later.

Zaitsev et al. $^{3}$ demonstrated $1.9 \mu \mathrm{m}$ InAs/InGaAs/InP QD lasers, which operated at $77 \mathrm{~K}$. In order to achieve laser operation at elevated temperatures, it is important to know the thermal evolution of the QD PL spectrum. Figure 3 shows the temperature dependent PL spectra of the QD sample, which could be measured up to $200 \mathrm{~K}$. At temperatures above about $100 \mathrm{~K}$ the lineshapes develop a pronounced asymmetry to the shorter wavelength side. The origin of this asymmetry can be explained by changes in the relative strengths of several underlying components of the PL spectra. These components were resolved by least-

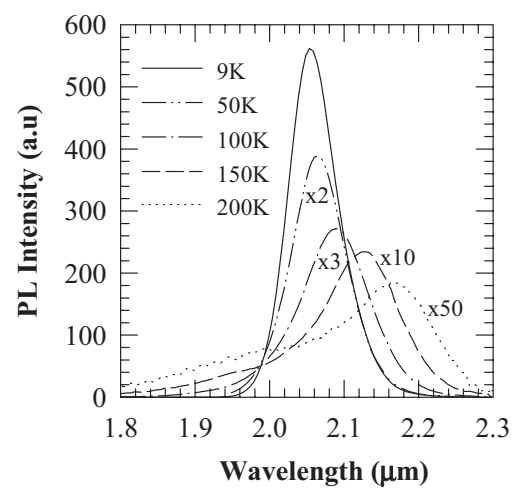

FIG. 3. The temperature dependent PL spectra of the InAs/InGaAs/InP QDs. The numbers next to each spectrum show the intensity magnification factors.

squares fitting with several Gaussian peaks, as already mentioned in the Fig. 2(a) example. Gaussian peaks are appropriate for fitting the PL transitions due to the spatial size distribution of the dots. ${ }^{14}$ All the spectra up to $100 \mathrm{~K}$ could be fitted satisfactorily using a single Gaussian component, as shown by the example in Fig. 4(a) for the 9 K PL spectrum. However, at $150 \mathrm{~K}$ we see a clear signature of another peak appearing on higher-energy side of the main feature. These two features were resolved by fitting two Gaussian components as shown in Fig. 4(b) by the dotted curves. The second

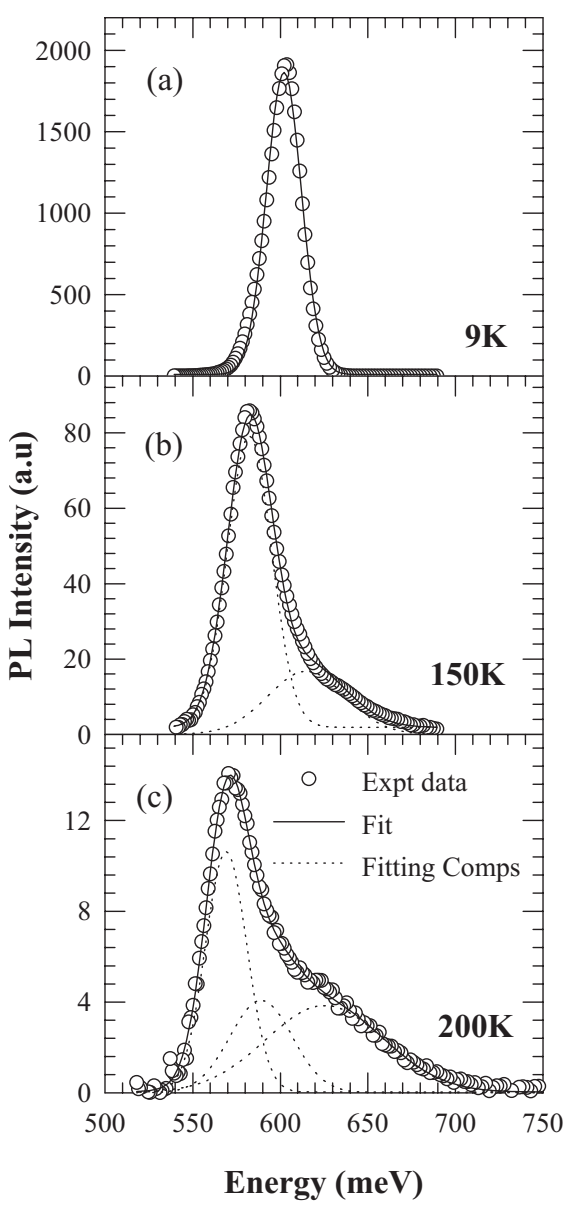

FIG. 4. Least-squares fits (full curves) of PL spectra at (a) $9 \mathrm{~K}$ with single Gaussian component, (b) $150 \mathrm{~K}$ with two Gaussian components, and (c) 200 $\mathrm{K}$ with three Gaussian components, with the individual components shown by the dotted curves. 


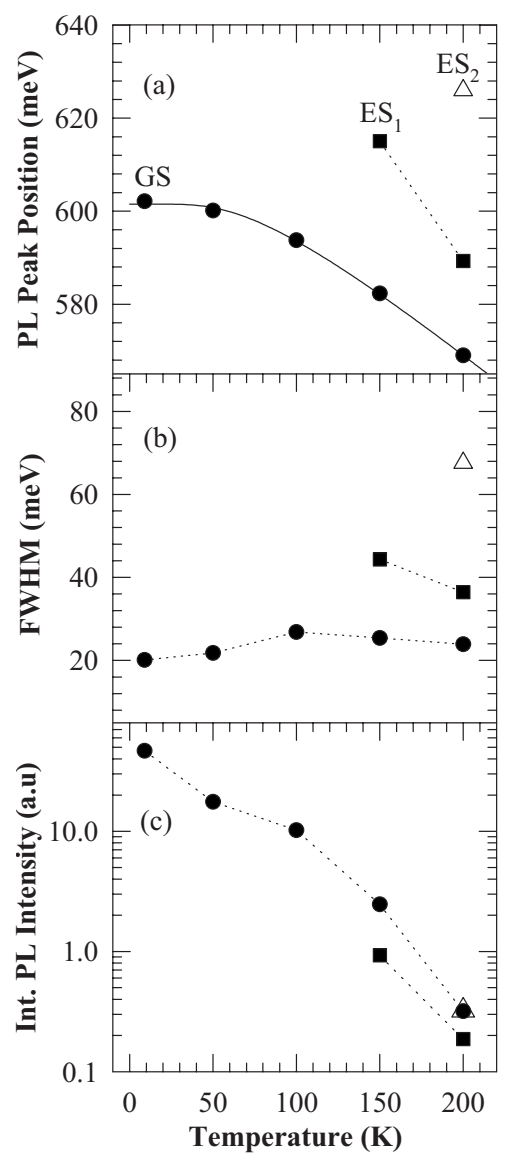

FIG. 5. Results of fitting the temperature dependent PL spectra, showing: (a) PL peak position, (b) FWHM, and (c) integrated PL intensity vs temperature. The circles, squares, and triangles are for transitions GS, $\mathrm{ES}_{1}$, and $\mathrm{ES}_{2}$, respectively. The full curve in (a) is a fit for transition energy GS with BE relation, i.e., Eq. (1) while the other lines are only guides to the eyes.

higher-energy peak can be related to the first ES ("ES,") of the QDs, which appears due to the thermal excitation of carriers from the ground to $\mathrm{ES}_{1}$. The analysis of $200 \mathrm{~K} \mathrm{PL}$ spectrum, however, was more troublesome. Here, a fit with two Gaussian components did not give a satisfactory fit, and the fitted peak energies did not relate well to those obtained from the fits at lower temperatures. However, if a fit with three Gaussians was performed [see Fig. 4(c)], with all parameters left entirely free to vary, the results were more physically realistic. Therefore, we contend that at $200 \mathrm{~K}$, a total of three QD transitions are now present in the PL spectrum, though they are certainly not all individually well resolved. This conclusion is supported by further evidence to be given later from the PR spectroscopy results. More detail on the thermal evolution of various electronic transitions is given in Fig. 5, where we plot the fitted PL peak position, full width at half maximum (FWHM), and integrated intensity of each of the PL Gaussian components, as a function of temperature. Figure 5(a) shows that the slope of the peak position of the second transition with temperature is steeper than that of the first transition. Similar characteristics of PL transition energies have earlier been observed by Lee et al. ${ }^{14}$ for InAs/GaAs QDs and can be explained on the basis of thermal escape of carriers from the high energy part of Gaussian distribution for the ESs, which subsequently re- duces their average energy at higher temperatures. ${ }^{14}$ The first feature is therefore identified as the ground state (GS) transition of the InAs/InGaAs/InP QDs, whereas the other two correspond to the first two ES transitions $\left(\mathrm{ES}_{1}\right.$ and $\mathrm{ES}_{2}$ ), as labeled in Fig. 5. It is interesting to note the large width of the $\mathrm{ES}_{1}$ and $\mathrm{ES}_{2}$ components as compared to that of the GS, e.g., in Fig. 4(c): at a given temperature, the width of the corresponding Gaussian peaks increases as their peak energy increases, with the highest-energy transition showing the maximum width. This is confirmed from Fig. 5(b) which shows that the fitted FWHM of the ES transitions increases with the energy of the transition, i.e., $\mathrm{FWHM}_{\mathrm{GS}}$ $<\mathrm{FWHM}_{\mathrm{ES}_{1}}<\mathrm{FWHM}_{\mathrm{ES}_{2}}$. Such a behavior is anticipated for InAs/InGaAs/InP QD structures due to the higher degeneracy of the high energy eigenstates that are also relatively "leaky" due to the graded low energy InGaAs barrier. Hence, due to their weaker quantum confinement, the ESs will be influenced more by any fluctuations in the barrier composition. Figure 5(b) shows that $\mathrm{FWHM}_{\mathrm{GS}}$ increases only weakly with temperature while $\mathrm{FWHM}_{\mathrm{ES}_{1}}$ decreases slightly from 150$200 \mathrm{~K}$. While there is likely to be some uncertainty in these values as derived from the fit, the weak (or negative) change with temperature suggests that the linewidth is strongly influenced by the inhomogeneous broadening of the QDs. The combination of inhomogeneous broadening, which decreases with heating (due to carrier thermalization), and homogeneous broadening, which increases with heating, leads to the observed overall weak temperature dependence of the linewidth. This has been observed in many QD systems and plays a pivotal role in the thermal characteristics of QD lasers. ${ }^{15}$ In Fig. 5(c) the integrated peak intensity of the GS can be seen to significantly decrease with increasing temperature. While some of this decrease at higher temperatures may be associated with carrier thermalization into the ESs, the overall decrease in quantum efficiency is most likely due to nonradiative Auger recombination, which increases strongly with heating and is known to be important in quantum well $(\mathrm{QW})$ and QD systems at these wavelengths. ${ }^{15,16}$

In Fig. 5(a), the temperature dependence of the fitted Gaussian peak energy of the GS transition is fitted with the Bose-Einstein (BE) relation given by Lautenschlager ${ }^{17}$

$$
E_{g}(T)=E_{B}-2 a_{B} /\left[\exp \left(\theta_{\mathrm{BE}} / T\right)-1\right],
$$

where the least-squares fit gives $E_{B}=602 \mathrm{meV}$ for the energy gap at $0 \mathrm{~K}, a_{B}=33.9 \mathrm{meV}$ represents the average strength of electron-phonon interaction, and $\theta_{\mathrm{BE}}=225 \mathrm{~K}$ corresponds to the mean phonon temperature. ${ }^{1,17}$ These values of fitting parameters are significantly larger than the reported values for bulk InAs $\left(a_{B}=20.7 \mathrm{meV}, \theta_{\mathrm{BE}}=147 \mathrm{~K}\right) .{ }^{18}$ However, the value of $a_{B}$ is significantly lower than those reported for InAs/InP QDs $\left(a_{B}=99 \mathrm{meV}\right) .{ }^{1} \mathrm{~A}$ small value of $a_{B}$ and a large value of $\theta_{\mathrm{BE}}$ indicate a less temperature sensitive eigenstate, as anticipated for coherently strained InAs QDs with strained InGaAs barriers.

To summarize the PL investigations, we obtained evidence for three transitions related to the InAs/InGaAs/InP QDs. Unfortunately, all these features are not simultaneously detectable in the PL at any given temperature. Hence, to obtain a more complete picture of the thermal evolution of 


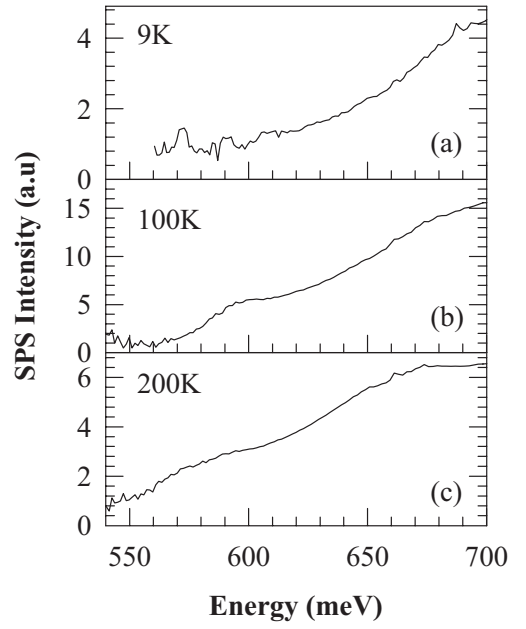

FIG. 6. Example SPS spectra of the InAs/InGaAs/InP QDs at three representative temperatures.

these QD features one would need to extrapolate the data available in Fig. 5(a). Such an extrapolation may not provide accurate results and is impossible here for transition $\mathrm{ES}_{2}$. Therefore, some complementary technique is needed such as measuring the absorption coefficient of the QD sample. However, common transmission measurements would yield nothing useful here because of the very low relative volume of the QD material. In such cases, SPS becomes a potentially useful tool since it can measure essentially very small values of the absorption coefficient such as in the case of InAs QDs ${ }^{19-22}$ or even ultrathin InAs QWs. ${ }^{23}$

This was the main reason we also applied the complementary SPS technique to the InAs/InGaAs/InP QD sample and indeed found a measurable signal at all temperatures. Figure 6 shows representative SPS spectra at three selected temperatures, showing that the overall magnitude of the SPS signal first increases between $\sim 9$ and $\sim 100 \mathrm{~K}$ but then subsequently drops. The initial increase in SPS signal with temperature can be associated with the thermal escape of charge carriers from the energy levels in the QDs, followed by their subsequent separation under the built-in electric field. For temperatures above $\sim 100 \mathrm{~K}$, nonradiative recombination processes begin to recapture the charge carriers, hence decreasing the SPS signal again. This is due to the fact that the SPS signal originates from those photogenerated carriers, which do not recombine either radiatively or nonradiatively.

To determine the QD transition energies $\left(E_{g}\right)$ in SPS, previous authors have fitted the experimental spectra with several Gaussian peaks. ${ }^{19,22}$ However, at first sight, the present SPS spectra appear to be entirely dominated by a rising absorption edge (see Fig. 6). The origin of this is not clear: it could be related to a complex signature emerging from the Urbach tail below the band gap of the graded and strained InGaAs barrier. Consequently, any QD features manifest themselves only weakly, as subtle features or changes in slope. In the case of the 9 K SPS spectrum in Fig. 6(a), the SPS signal is very weak and noisy at low energies but exhibits a clear onset and rise out of this noise near a threshold energy of $\sim 605 \mathrm{meV}$, which is in reasonable agreement with the $9 \mathrm{~K}$ PL measurement of the GS energy.

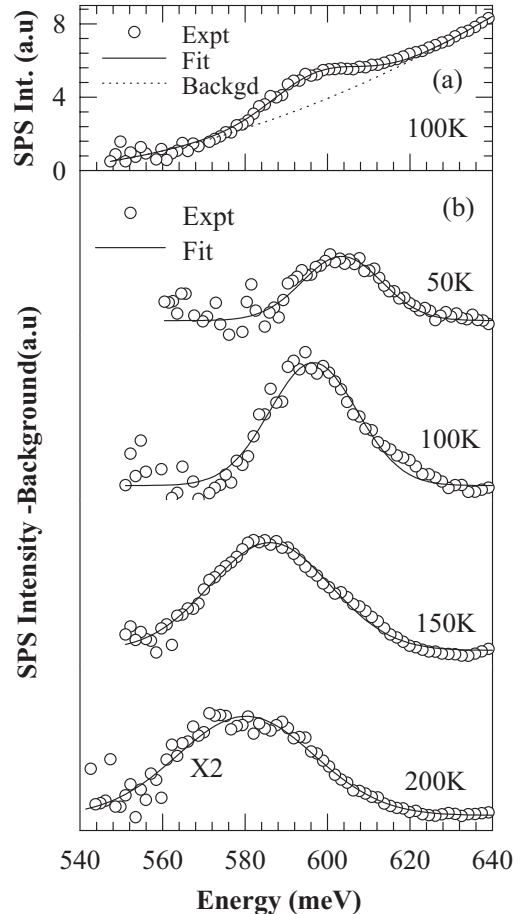

FIG. 7. (a) Least-squares fits of the SPS spectrum at $100 \mathrm{~K}$ with a single Gaussian peak along with Urbach tail. (b) The same fitting exercise at four temperatures shown with the Urbach tail removed for clarity in presentation.

At higher temperatures the SPS spectra begin to show somewhat clearer evidence of a single weak peaklike feature superimposed on the Urbach tail, as may be seen, for example, in Fig. 6(b) at $100 \mathrm{~K}$ near $\sim 590 \mathrm{meV}$. In order to extract more reliable energies for these subtle peaks, it was necessary to represent the underlying rising absorption background with some suitable functional form. Though an Urbach tail is usually assumed to have an exponential form below the respective band gaps, ${ }^{24-26}$ this is evidently not generally the case here, as may be seen from Fig. 6. Instead, it was found convenient to parametrize this background with a simple quadratic term, which gave a reasonably accurate fit over a limited energy range, while the peak feature was fitted with a single additive Gaussian peak on top of the quadratic form. An example of this fitting procedure is shown in Fig. 7(a) for $100 \mathrm{~K}$ SPS data. In Fig. 7(b), we again plot the SPS data along with fitted curves at four temperatures, but, for the purposes of clarity, the quadratic background has now been removed in order to emphasize the remnant SPS peak and its Gaussian fit. ${ }^{19,22}$ It is worth noting that we were also able to obtain reasonable fits to the SPS peaks using a Lorentzian lineshape, appropriate to excitonic absorption profiles in quantum-confined systems, ${ }^{27}$ but this gave almost identical results to the Gaussians for the fitted peak energies. The fitted SPS Gaussian peak energies are plotted in Fig. 8 along with the earlier PL transition energy values, which, as may be seen, are in reasonable agreement, except at $200 \mathrm{~K}$. Here, and at higher temperatures, the SPS spectra again became weaker [as can be seen in Fig. 7(b), where the $200 \mathrm{~K}$ spectrum is multiplied by a factor of 2]. It can also be seen from Fig. 7(b) that the fit at $200 \mathrm{~K}$ is poorer than at lower temperatures. In fact, we are unable to determine unambiguously 


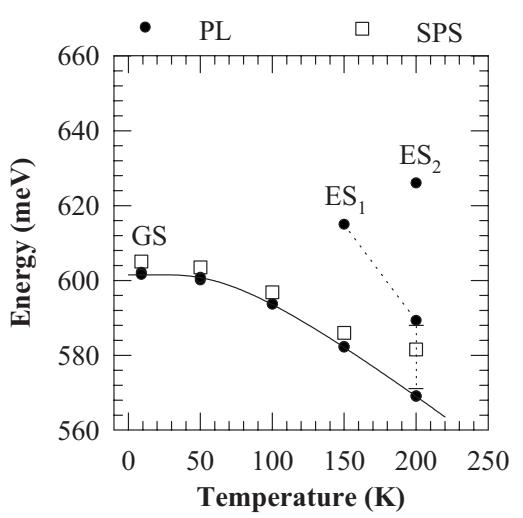

FIG. 8. A comparison of the QD transition energies determined by the PL and SPS measurements as a function of temperature. The dotted line is only a guide to the eyes. The large error bar for the SPS result at $200 \mathrm{~K}$ is due to uncertainty in fitting. The errors in the other SPS results are smaller than the symbol size. The full curve is as in Fig. 5(a).

whether the 200 K SPS peak feature constitutes a singlet or a barely resolved doublet: certainly a better fit could be achieved by assuming a doublet. Consequently, the large error bar in the $200 \mathrm{~K} \mathrm{SPS}$ result in Fig. 8 reflects this statistical uncertainty: it may be seen, however, that the SPS error bar satisfactorily embraces the two low-energy PL results. At lower temperatures the estimated errors in the SPS energies are smaller than the symbol size in Fig. 8. The FWHMs of the fitted GS SPS peaks were all larger than the corresponding PL widths [see Fig. 5(b)], increasing steadily from $\sim 23 \mathrm{meV}$ at $50 \mathrm{~K}$ to $\sim 39 \mathrm{meV}$ at $200 \mathrm{~K}$.

$\mathrm{PR}$ is another well-known versatile technique, which can be applied to study QD transitions. ${ }^{1,14,19,28-30}$ However, it can be quite difficult to get clear QD PR signatures, due to the small QD volume and consequential small signal amplitudes. There are several PR studies of either InAs/GaAs QDs $^{14,19,28-30}$ or InAs/InP QDs. ${ }^{1}$ However, we are not aware of any previous PR investigations of InAs/InGaAs/InP QDs emitting at $\sim 2 \mu \mathrm{m}$. Here, we were able to record clean PR spectra over a wide range of temperatures, as shown by the examples in Fig. 9. The strongest feature seen at the highest energies $(>\sim 650 \mathrm{meV})$ corresponds to the strained InGaAs barrier grown on the InP buffer. To confirm this we calculated the expected InGaAs barrier band gap, taking strain effects into account, as described by Pollak. ${ }^{31}$ The material parameters for InAs and GaAs were taken from the literature. ${ }^{32}$ For $\mathrm{In}_{0.72} \mathrm{Ga}_{0.28}$ As grown on InP (see Table I), we obtain a band gap of $\sim 671 \mathrm{meV}$ at $0 \mathrm{~K}$, which lies within experimental uncertainty of the measured feature at $\sim 680 \mathrm{meV}$ at $9 \mathrm{~K}$ (see Fig. 9 and also fitting results described next). To match this measured strained barrier band gap of $\sim 680 \mathrm{meV}$ an alloy composition of $\mathrm{In}_{0.707} \mathrm{Ga}_{0.293} \mathrm{As}$ is required, which is in reasonable agreement with the planned nominal composition within the tolerances of MOVPE growth. ${ }^{12}$

For an accurate determination of the transition energy of interband QD electronic transitions in PR measurements, we fit the spectra with a sum of Aspnes line shape functions ${ }^{33}$

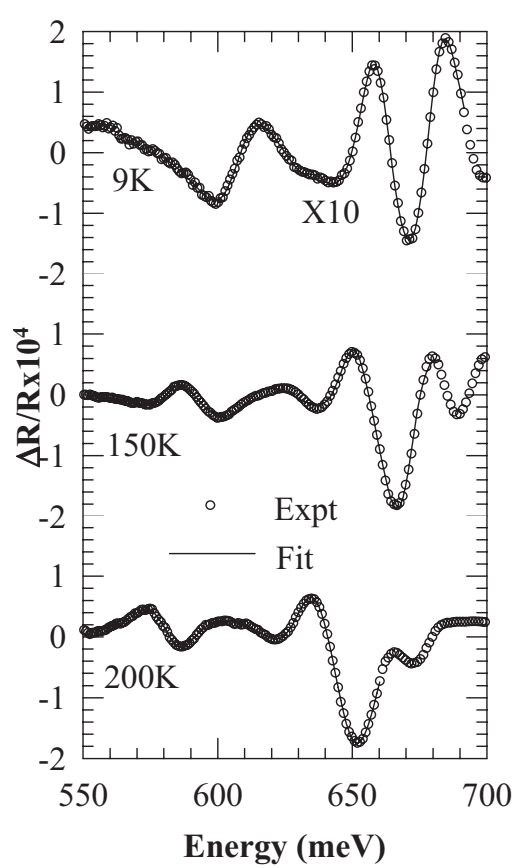

FIG. 9. Example PR spectra of the InAs/InGaAs/InP QDs at three representative temperatures. The full curves show FDLL fits using Aspnes line shape function, i.e., Eq. (2) with four oscillators.

$$
\frac{\Delta R}{R}=\sum_{j=1}^{n} \operatorname{Re}\left\{A_{j} e^{\left.i \theta_{j} /\left(E-E_{0 j}+i \Gamma_{j}\right)^{m_{j}}\right\}}\right.
$$

where $n$ is the number of critical point transition features, $j$ is the critical point index, $A_{j}$ is an amplitude, $\theta_{j}$ is a phase angle, $E_{0 j}$ is the critical point energy, $\Gamma_{j}$ is a broadening parameter, and $m_{j}$ is an exponent that depends on the dimensionality of the critical point transition. For example, with $m_{j}=2$ the Aspnes function corresponds to the first derivative of a Lorentzian line shape (FDLL), which is often used to fit experimental PR spectra of excitonic transitions. ${ }^{34}$ Figure 9 shows the excellent fits obtained with Eq. (2) with $m_{j}=2$ and $n=4$ at the three temperatures, while Fig. 10 shows a decomposition of the $150 \mathrm{~K}$ fit into the four component oscillators. Figure 11 shows the final outcome of fitting the PR spectra recorded from 9 to $200 \mathrm{~K}$, together with the previous PL results, for the purposes of comparison. It is obvious that the

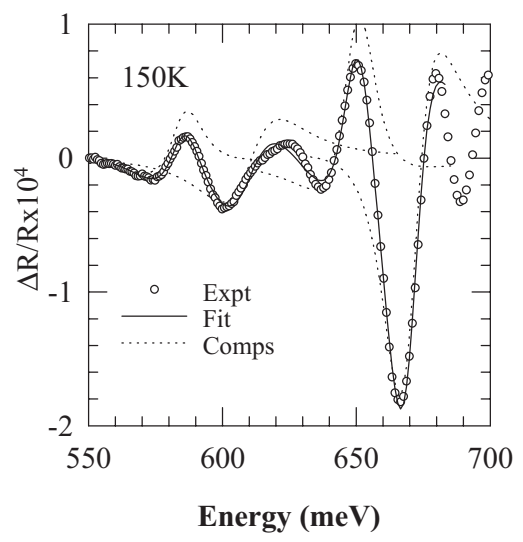

FIG. 10. Detail of the fit shown in Fig. 9 for the $150 \mathrm{~K}$ PR spectrum. The four individual FDLL components are shown by the dotted curves. 


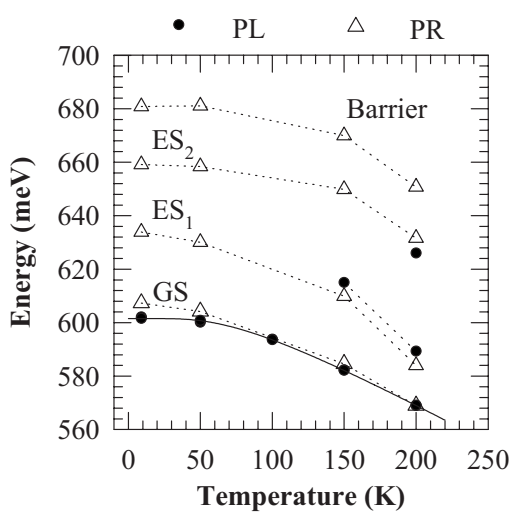

FIG. 11. A comparison of the transition energies determined by the PL and PR measurements as a function of temperature. The dotted lines are only a guide to the eyes. The full curve is as in Fig. 5(a).

variation with temperature of the fitted PR energy of feature "GS" matches very well with the corresponding PL results for the GS transition. Transitions $\mathrm{ES}_{1}$ and $\mathrm{ES}_{2}$ are related to ES transitions of the InAs/InGaAs/InP QDs whereas the highest-energy transition originates from the strained InGaAs barrier, as explained earlier. The fitted FWHMs of the four PR features were comparable to those shown in Fig. 5(b) for the PL, ranging from $\sim 20$ to $\sim 40 \mathrm{meV}$, with a similar general weak increase with heating. At temperatures below $\sim 150 \mathrm{~K}$ the three fitted PR QD transition energies have a nearly equal spacing (of $\sim 26 \mathrm{meV}$ ): equally spaced transition energies are consistent with the expectations of a twodimensional parabolic harmonic oscillator confinement potential, as is often proposed in the literature. ${ }^{22,28}$

It is of considerable interest to compare the temperature dependence of all the QD electronic transitions as measured by the three complementary spectroscopic techniques. There does not appear to be any such comparison for QD features in the literature, using complementary measurements performed indubitably on the same sample spot. Figure 12 shows the summary of all the virtually simultaneous complementary spectroscopic measurements performed on the InAs/ InGaAs/InP QDs sample under essentially identical condi-

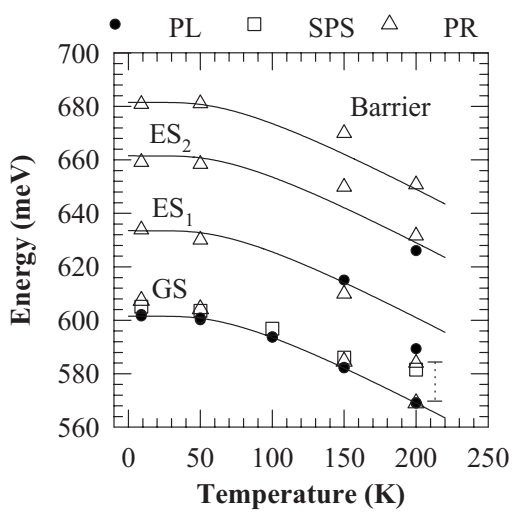

FIG. 12. A summary of the full set of results showing the QD transition energies determined by the three virtually simultaneous complementary PL, PR, and SPS techniques. The transition energy vs temperature results are fitted using BE relation, i.e., Eq. (1) as shown by the solid curves. The uncertainties in the fitted energies are all smaller than or comparable to the sizes of the respective symbols, except in the case of the $200 \mathrm{~K}$ SPS result where the large dotted error bar is due to uncertainty in fitting. tions. In Fig. 12, all the available observed transition energies, from GS to the barrier from all three techniques, are shown compared to $\mathrm{BE}$ curves using the same set of parameters [see Eq. (1)] as obtained earlier for transition GS in the PL measurements [see Fig. 5(a)] except with different appropriate fitted values for $E_{B}(602,634,662$, and $682 \mathrm{meV}$ for transitions GS to the barrier, respectively). Clearly, the agreement shown in Fig. 12 between the BE curves and the experimental measurements is generally satisfactory.

\section{CONCLUSION}

An accurate determination of the QD transitions has been obtained for InAs/InGaAs/InP QDs by performing virtually simultaneous PL, PR, and SPS measurements. This ensures that the three spectroscopic techniques map the same sample spot under almost identical conditions. We could measure the PL spectrum up to $200 \mathrm{~K}$. It is observed that, at a given temperature, the FWHMs of the QD transitions observed in the PL spectrum increase steadily from the GS transition through to the higher ESs. This can be understood in terms of the impact of compositional fluctuations in the graded InGaAs barrier, which would affect the ESs more strongly because of their weaker quantum confinement. Temperature dependent PL, PR, and SPS measurements provided a description of the systematic thermal evolution of ground and ES transition energies. However, all the QD transitions were not seen altogether at any given temperature in either the PL or SPS measurements: in the PL we observe a maximum of three QD transitions (the ground and two ESs) but only at $200 \mathrm{~K}$, while in the SPS, only a broad GS QD peak is unambiguously detectable at all temperatures, after accounting for a strong underlying Urbach tail arising from the InGaAs barrier. In contrast, the PR technique could resolve the full set of ground and two ES transitions of the InAs QDs, as well as the InGaAs barrier energy at all studied temperatures.

\section{ACKNOWLEDGMENTS}

One of the authors (T.K.S) acknowledges the British Council, New Delhi for providing the financial support for this work under the UKIERI fellowship scheme. X.Tang thanks A*STAR and NTU, Singapore for funding the growth program. We also thank Natasha Fox for her help in the acquisition of some of the spectra presented.

${ }^{1}$ H. Chouaib, N. Chauvin, C. B. Chevallier, C. Monat, P. Regreny, and M. Gendry, Appl. Surf. Sci. 253, 90 (2006).

${ }^{2}$ B. Salem, T. Benyattou, G. Guillot, C. B. Chevallier, G. Bremond, C. Monat, G. Hollinger, and M. Gendry, Phys. Rev. B 66, 193305 (2002).

${ }^{3}$ S. V. Zaitsev, N. Y. Gordeev, V. I. Kopchatov, V. M. Ustinov, A. E. Zhukov, A. Y. Egorov, A. R. Kovsh, and P. S. Kop'ev, Jpn. J. Appl. Phys., Part 1 38, 601 (1999).

${ }^{4}$ Z. Yin and X. Tang, Solid-State Electron. 51, 6 (2007).

${ }^{5}$ Z. Yin, X. Tang, W. Liu, D. Zhang, and A. Du, J. Appl. Phys. 100, 033109 (2006).

${ }^{6}$ O. J. Glembocki and B. V. Shanabrook, Spectroscopy of Semiconductors, Semiconductors and Semimetals, edited by D. G. Seiler and C. G. Littler (Academic, San Diego, CA, 1992), Vol. 36, Chap. 4, pp. 239-252.

${ }^{7}$ T. J. C. Hosea, Thin Solid Films 450, 3 (2004).

${ }^{8}$ L. Kronik and Y. Shapira, Surf. Sci. Rep. 37, 1 (1999).

${ }^{9}$ Y. S. Huang and F. H. Pollak, Phys. Status Solidi A 202, 1193 (2005).

${ }^{10}$ S. Datta, S. Ghosh, and B. M. Arora, Rev. Sci. Instrum. 72, 177 (2001).

${ }^{11}$ T. K. Sharma, S. Porwal, R. Kumar, and S. Kumar, Rev. Sci. Instrum. 73, 
1835 (2002).

${ }^{12}$ T. K. Sharma, B. M. Arora, S. Kumar, and M. R. Gokhale, J. Appl. Phys. 91, 5875 (2002).

${ }^{13}$ G. D. Gilland, Mater. Sci. Eng., R. 18, 99 (1997).

${ }^{14}$ J. R. Lee, C. R. Lu, W. I. Lee, and S. C. Lee, Physica E (Amsterdam) 25 , 562 (2005).

${ }^{15}$ I. P. Marko, N. F. Masse, S. J. Sweeney, A. D. Andreev, A. R. Adams, N. Hatori, and M. Sugawara, Appl. Phys. Lett. 87, 211114 (2005).

${ }^{16}$ B. N. Murdin, A. R. Adams, and S. J. Sweeney, Mid-infrared Semiconductor Optoelectronics, Springer Series in Optical Sciences (Springer, New York, 2006)

${ }^{17}$ P. Lautenschlager, M. Garriga, and M. Cardona, Phys. Rev. B 36, 4813 (1987).

${ }^{18}$ R. Pässler, Phys. Rev. B 66, 085201 (2002).

${ }^{19}$ B. Q. Sun, Z. D. Lu, D. S. Jiang, J. Q. Wu, Z. Y. Xu, Y. Q. Wang, J. N. Wang, and W. K. Ge, Appl. Phys. Lett. 73, 2657 (1998).

${ }^{20}$ J. Toušková, E. Samochin, J. Toušek, J. Oswald, E. Hulicius, J. Pangrác, K. Melichar, and T. Šimeček, J. Appl. Phys. 91, 10103 (2002).

${ }^{21}$ H. Pettersson, L. Landin, Y. Fu, M. Kleverman, M. Borgstrom, W. Seifert, and L. Samuelson, Microelectron. J. 36, 227 (2005).

${ }^{22}$ J. Bhattacharyya, S. Ghosh, S. Malzer, G. H. Döhler, and B. M. Arora, Appl. Phys. Lett. 87, 212101 (2005).
${ }^{23}$ S. D. Singh, S. Porwal, T. K. Sharma, and K. C. Rustagi, J. Appl. Phys. 99, 063517 (2006)

${ }^{24}$ F. Urbach, Phys. Rev. 92, 1324 (1953).

${ }^{25}$ C. W. Greeff and H. R. Glyde, Phys. Rev. B 51, 1778 (1995).

${ }^{26}$ D. B. Tran Thoai and H. Hang, Phys. Rev. B 47, 3574 (1993).

${ }^{27}$ C. Tanguy, P. Lefebvre, H. Mathiev, and R. J. Elliot, J. Appl. Phys. 82, 798 (1997).

${ }^{28}$ G. L. Rowland, T. J. C. Hosea, S. Mallik, D. Childs, and R. Murray, Appl. Phys. Lett. 73, 3268 (1998).

${ }^{29}$ C. M. Lai, F. Y. Chang, C. W. Chang, C. H. Kao, H. H. Lin, G. J. Jan, and J. Lee, Appl. Phys. Lett. 82, 3895 (2003).

${ }^{30}$ P. Hazdra, J. Voves, J. Oswald, K. Kuldova, A. Hospodkova, E. Hulicius, and J. Pangrac, Microelectron. J. 39, 1070 (2008).

${ }^{31}$ F. H. Pollak, in Strained-Layer Superlattices: Physics, Semiconductors and Semimetals, edited by T. P. Pearsall (Academic, San Diego, CA, 1990), Vol. 32, Chap. 2.

${ }^{32}$ I. Vurgaftman, J. R. Meyer, and L. R. Ram-Mohan, J. Appl. Phys. 89, 5815 (2001).

${ }^{33}$ D. E. Aspnes, in Handbook on Semiconductors, edited by T. S. Moss (North Holland, New York, 1980), Vol. 2, p. 109, and references therein.

${ }^{34}$ Y. S. Huang, H. Qiang, F. H. Pollak, G. D. Pettit, P. D. Kirchner, J. M. Woodall, H. Stragier, and L. B. Sorensen, J. Appl. Phys. 70, 7537 (1991). 